\title{
A Volume Holographic Sol-Gel Material with Large Enhancement of Dynamic Range by Incorporation of High Refractive Index Species**
}

\author{
By Francisco del Monte,* Oscar Martínez, José A. Rodrigo, María L. Calvo, and Pavel Cheben
}

The development of an optimum recording material remains one of the principal challenges in the area of holographic data storage. ${ }^{[1]}$ Holographic photopolymers, first reported in the late 1960 s by Close et al., ${ }^{[2]}$ are attractive candidates for applications in data storage because they can be designed to store permanent phase holograms with a large dynamic range and high photosensitivity, and, unlike other holographic recording materials, they do not require complicated holographic development processes. Photopolymers typically consist of one or more acrylic monomers and a photoinitiator complex, all dispersed within a polymer matrix, also referred to as a binder ${ }^{[3]}$ Holograms are stored in photopolymers as a permanent spatial modulation of refractive index within the bulk of the material, which is formed in response to the interference pattern of light and dark fringes produced by two laser beams intersecting in the material. The photoinduced polymerization of the acrylic monomer in the light regions of the interference pattern produces a refractive index increase in the illuminated regions compared to the dark regions. A large refractive index difference $\Delta n$ between these two regions is desired to maximize the dynamic range, or the data storage capacity, of the material. A combination of one or more large refractive index monomers (e.g., aromatic monomers) with a low refractive index binder is known to be advantageous for maximizing the refractive index modulation $\Delta n$. However, obtaining a permanent hologram requires another mechanism (e.g., the Colburn and Haines mechanism) in addition to monomer polymerization in the illuminated re-

[*] Dr. F. del Monte

Institute for Materials Science, Madrid-ICMM

Spanish Research Council-CSIC

Cantoblanco, 28049 Madrid (Spain)

E-mail: delmonte@icmm.csic.es

Dr. O. Martínez, J. A. Rodrigo, Prof. M. L. Calvo

Optical Department, Faculty of Physical Sciences

University Complutense, Madrid

28040 Madrid (Spain)

Dr. P. Cheben

Institute for Microstructural Sciences

National Research Council of Canada

Ottawa K1A OR6 (Canada)

[**] This work was supported by MEC under the projects MAT2003 02718 and TIC2002-1846, and by CM under the project S0505/PPQ0316. We gratefully acknowledge Carmen Munuera and Prof. Carmen Ocal for technical assistance and discussions concerning STM. Supporting Information is available online from Wiley InterScience or from the authors. gions of the interference pattern (Scheme 1, left). ${ }^{[4]}$ Otherwise, the hologram would be erased upon homogeneous incoherent exposure (often used to stabilize holograms recorded in photopolymers) due to the polymerization of the remaining monomer in dark regions of the interference pattern (see Supporting Information for further details).

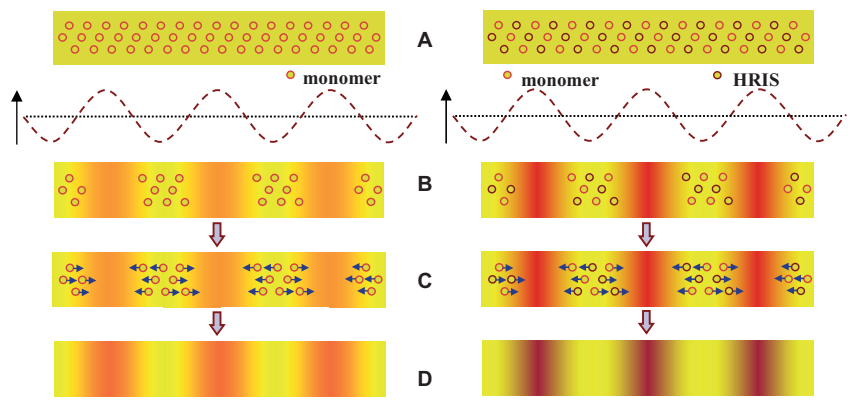

Scheme 1. Formation of a permanent spatial modulation of refractive index within the volume of a photopolymerizable material by single-monomer (left) and monomer-and-HRIS (right) diffusion following the diffraction pattern generated by interference of two coherent laser beams (see Supporting Information for further explanations). Lighter colors (i.e., yellow) represent low refractive index fringes, and darker colors (i.e., orange for monomer and brown for HRIS) high refractive index fringes.

Achieving refractive index modulations $\Delta n$ larger than ca. $5 \times 10^{-3}$ has proven to be difficult in photopolymerizable compositions, ${ }^{[5]}$ in part because of the limited diffusion of monomer molecules in binders and also because of relatively limited refractive index differences between the available monomers and binders. Attempts to improve the refractive index modulation have been made by incorporating high refractive index species (HRIS), such as silica and titania nanoparticles, in addition to the regular photopolymerizable monomer, ${ }^{[6]}$ yielding volume holographic gratings with refractive index modulations of up to $1.5 \times 10^{-2}$ (Scheme 1 , right). ${ }^{[6 \mathrm{c}]}$ However, this interesting approach results in a large scattering (ca. $15 \%$ for samples of $15 \mu \mathrm{m}$ thickness), apparently due to nanoparticle agglomeration after diffusion, which is a serious limitation for applications demanding low levels of scattering, such as holographic data storage.

In this communication, we also propose the incorporation of HRIS (in addition to the photopolymerizable monomer 2phenoxyethyl acrylate, POEA), in a volume holographic recording material. However, in order to minimize scattering, 
such inorganic species are introduced at a molecular level (here we chose zirconium isopropoxide, $\left.\mathrm{Zr}\left(\mathrm{O}^{i} \mathrm{Pr}\right)_{4}\right)$, rather than in the form of nanoparticles. Actually, $\mathrm{Zr}\left(\mathrm{O}^{i} \mathrm{Pr}\right)_{4}$ has been incorporated in sol-gel materials for optical applications demanding high refractive index modulation (e.g., gradient index (GRIN) lenses). ${ }^{[7]}$ The use of $\mathrm{Zr}\left(\mathrm{O}^{i} \mathrm{Pr}\right)_{4}$ requires its hydrolysis kinetics to be slowed down through the formation of complexes with chelating agents (e.g., methacrylic acid (MA) or 2,4-pentadienone). In this case, we chose MA because, upon photopolymerization, concentration-gradientdriven diffusion transport of the whole MA: $\mathrm{Zr}$ complex is expected to occur. Further components of the holographic materials are POEA as the second photopolymerizable monomer and bis $\left(\mu^{5}-2,4\right.$-cyclopentadien-1-yl)-bis[2,6-difluoro-3(1H-pyrrol-1-yl)phenyl]titanium (IRGACURE-784) as a freeradical-generating photoinitiator. The binder of choice is a nanoporous hybrid silica glass similar to those described since the seminal works by Chandross et al.,${ }^{[8]}$ Borelli and Morse, ${ }^{[9]}$ and Sukhanov et al., ${ }^{[10]}$ and the first photopolymerizable solgel volume holographic material by Cheben et al. ${ }^{[11]}$ POEA is the acrylic monomer of choice, given its already studied behavior in a photopolymerizable silica glass. ${ }^{[12]}$

The expected improvement in the holographic performance of our material resides in the ability of the MA:Zr complex (HRIS) to diffuse upon inhomogeneous illumination. To experimentally confirm the contribution to refractive index modulation due to HRIS, we studied two types of compositions: one with and one without zirconium isopropoxide, denoted samples $\mathrm{Zr} 1$ and $\mathrm{Zr} 0$, respectively, in the text. Samples $\mathrm{Zr} 1$ and $\mathrm{Zr} 0$ were chosen to have nearly identical thickness (ca. $35 \mu \mathrm{m})$ to facilitate the comparison of their holographic performance. The holograms were recorded five days after gelation.

To characterize the holographic performance of two compositions, plane gratings were recorded by two mutually coherent collimated writing beams of wavelength $532 \mathrm{~nm}$ (see the Experimental section for details). Diffraction efficiency was measured during and after the holographic exposure by a non-actinic probe beam from a He-Ne laser of $632.8 \mathrm{~nm}$ wavelength. The maximum diffraction efficiency $\eta$ (where $\eta=P_{-1} / P_{0}, P_{-1}$ is the power in the -1 st diffraction order of the probe beam, and $P_{0}$ is the power in the probe beam incident on the sample) for samples $\mathrm{Zr} 0$ and $\mathrm{Zr} 1$ was reached for exposures of 1.8 and $3.0 \mathrm{~J} \mathrm{~cm}^{-2}$, respectively (Fig. 1a). The grating angular selectivity half-width $(\theta)$ was obtained from the measurement of the diffraction efficiency as a function of angular deviation from the Bragg condition (Fig. 1b). The refractive index modulation amplitude $\Delta n$ and the effective thickness of the grating $\left(t_{\text {eff }}\right)$ were calculated from the diffraction efficiency and the angular selectivity curve using Kogelnik's coupled wave theory. ${ }^{[13]}$ The photosensitivity ${ }^{[14]}$ was calculated as the ratio between the square root of the diffraction efficiency and the product of the exposure $(E)$ and the interference fringe visibility $(v): S=\eta^{1 / 2} /(E v)$.

The composition of $\mathrm{Zr} 0$ is similar to that reported recently for a highly efficient photopolymerizable glass comprising POEA monomer and IRGACURE-784 photoinitiator dis-
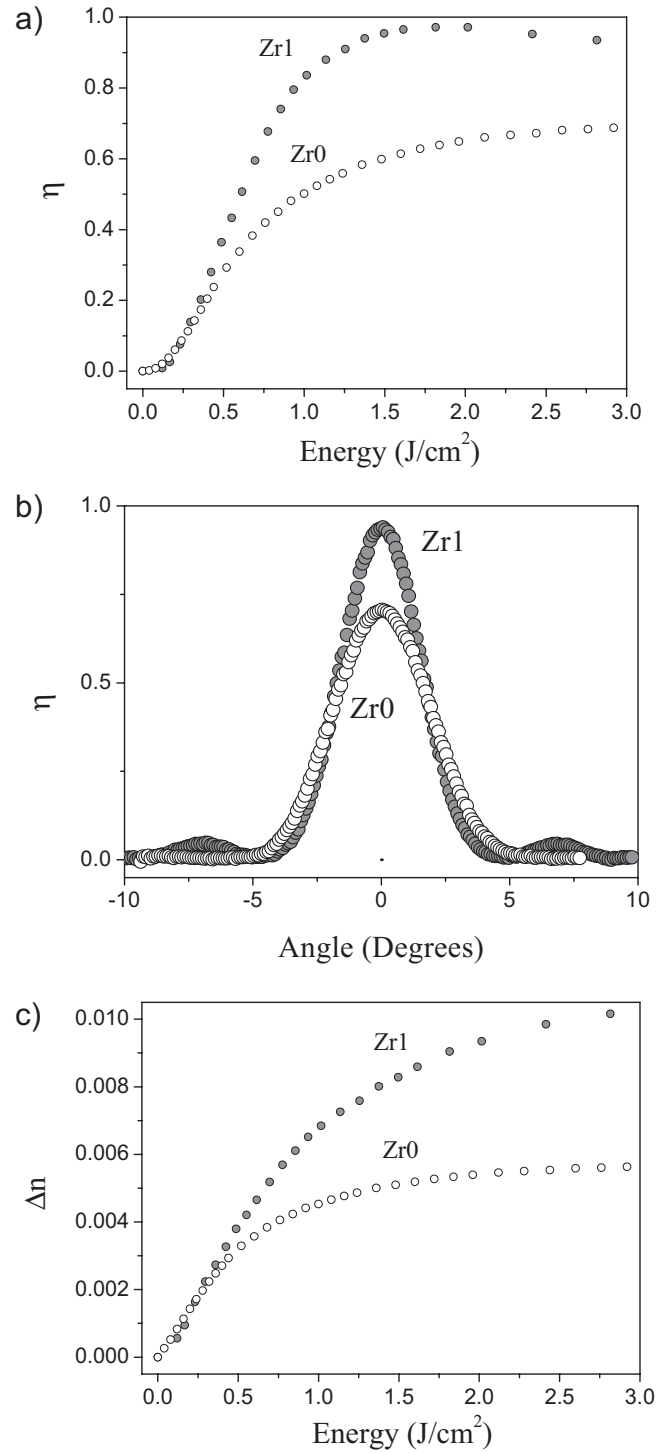

Figure 1. a) Diffraction efficiencies versus energy exposure in samples $\mathrm{ZrO}$ and $\mathrm{Zr} 1$. b) Angular selectivity curves for samples $\mathrm{ZrO}$ and $\mathrm{Zr} 1$. Values of the full width at half maximum are $\theta_{\mathrm{HW}}=4.40^{\circ}$ and $3.64^{\circ}$ for $\mathrm{ZrO}$ and $\mathrm{Zr} 1$ concentrations, respectively. c) Energy exposure dependence of the grating refractive index modulation in samples $\mathrm{ZrO}$ and $\mathrm{Zr}$. The thickness of both samples is $35 \mu \mathrm{m}$.

persed in a silica gel host. ${ }^{[12]}$ As shown in Figure 1c, incorporation of HRIS in the host markedly improves the refractive index modulation of the material, reaching $\Delta n \sim 10^{-2}$ compared to $\Delta n \sim 5.6 \times 10^{-3}$ in the sample without the HRIS (the exposure energy is ca. $2.8 \mathrm{~J} \mathrm{~cm}^{-2}$ ). In sample $\mathrm{Zr} 1$, maximum efficiency of $97 \%$ is reached for $E \sim 1.8 \mathrm{~J} \mathrm{~cm}^{-2}$; further increase in exposure leads to a slight decrease in $\eta$ (see Fig. 1a) due to refractive index over-modulation, as is expected from coupled-wave theory. ${ }^{[13]}$ In sample $\mathrm{Zr} 0$, maximum diffraction efficiency of $69 \%$ is achieved for $E \sim 2.92 \mathrm{~J} \mathrm{~cm}^{-2}$, where a plateau is reached as the index modulation versus exposure response saturates (Fig. 1a). The achievement of index modulation of ca. $10^{-2}$ in sample $\mathrm{Zr} 1$ is 
remarkable, and this value is among the best obtained in a holographic photopolymerizable composition of similar thickness. ${ }^{[5]}$ At the same time, photosensitivity is similar to POEAbased photopolymerizable glasses, ${ }^{[12]}$ and high diffraction efficiencies close to the theoretical $100 \%$ limit can be obtained even for relatively thin samples with a Klein-Cook parameter $Q \sim 20$. The $Q$ value confirms the volume (Bragg) nature of these gratings, with insignificant contribution of surface modulation, as is also confirmed by the scanning force microscopy (SFM) image shown in Figure 2. Note the low value of the root mean square (RMS) of surface modulation of sample $\mathrm{Zr1}$. Furthermore, the effective thickness of the sample (e.g., $35 \mathrm{~mm}$ ) is in good agreement with the grating thickness calculated from Kogelnik's theory for volume gratings.

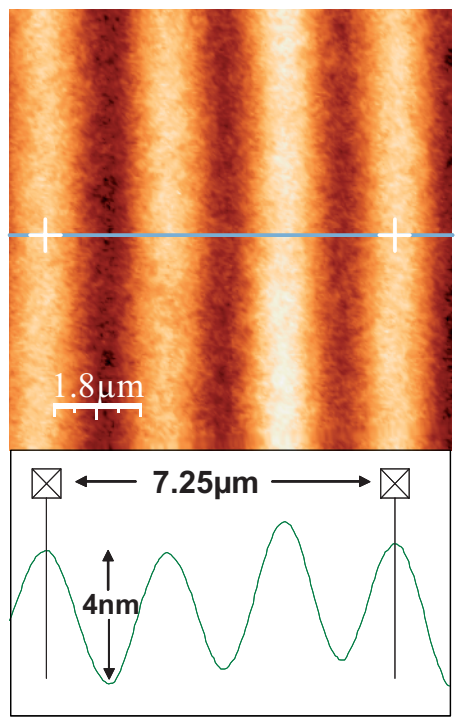

Figure 2. SFM tomography of sample $\mathrm{Zr} 1$.

Additional confirmation of the MA: $\mathrm{Zr}$ complex diffusion was obtained by performing energy dispersive X-ray analysis (EDX) on various samples with holographically recorded gratings. Figure 3 shows a scanning electron microscopy (SEM) image of the volume grating created in the material upon interferometric exposure. In this case and owing to measurement limitations of the SEM equipment, namely the smallest spot diameter for EDX analysis of ca. $2 \mu \mathrm{m}$, the grating spatial frequency was 100 lines $\mathrm{mm}^{-1}$, that is, a grating period of $10 \mu \mathrm{m}$. The $\mathrm{Zr} / \mathrm{Si}$ elemental ratio measured by EDX shows that HRIS concentration increases from lighter to darker fringes. Thus, while the $\mathrm{Zr} / \mathrm{Si}$ atomic ratio on average is 0.11 , in agreement with the molar ratios used in sample preparation (see Experimental section), the $\mathrm{Zr} / \mathrm{Si}$ elemental ratio in the darker fringes is about $10 \%$ higher than in the lighter ones ( 0.115 vs. 0.105 , respectively), for any spatial selection of the spot for EDX analysis, that is, nearer the upper surface, nearer the substrate, or in between them (see yellow circles in Fig. 3). This confirms a good grating homogeneity, as already discussed above by comparing the grating effective thickness

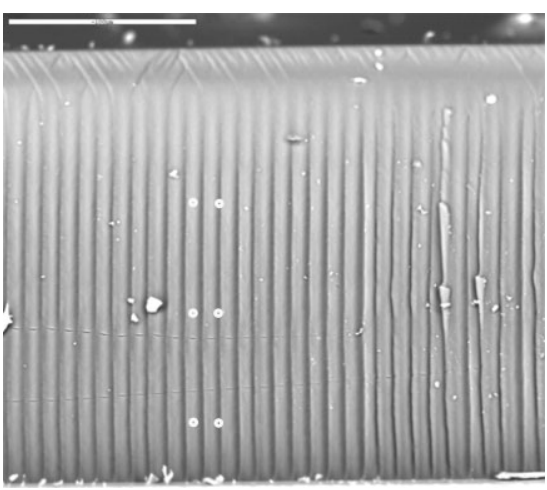

Figure 3. SEM image of a volume phase grating of spatial frequency 100 lines $\mathrm{mm}^{-1}$ holographically recorded in sample $\mathrm{Zr} 1$ with $250 \mu \mathrm{m}$ thickness. EDX analysis was performed at the marked white circles. Scale bar is $100 \mu \mathrm{m}$.

calculated from coupled wave theory with the physical grating thickness measured by SEM. The Zr gradient obtained from EDX for these low spatial frequency gratings (100 lines $\left.\mathrm{mm}^{-1}\right)$ is a conservative estimate of the concentration gradient compared to gratings with a higher spatial frequency, such as those recorded in samples $\mathrm{Zr} 0$ and $\mathrm{Zr} 1$ (500 lines $\mathrm{mm}^{-1}$ ). Note that, for the latter, diffusion of HRIS must be favored because of a fivefold reduction in dimensional range compared to the sample analyzed by EDX.

Besides holographic performance, incorporation of HRIS in molecular form is crucial for the reduction of coherent and incoherent scattering noise, which is one of the fundamental challenges in holographic data storage applications. Scattering originates in surface and volume material imperfections; the latter are of particular concern in materials with large thickness. Scattered light interfering with incident beams forms noise holograms, hence reducing dynamic range and deteriorating crosstalk in multiplexed holograms. To study scattering in our material, we performed two independent experiments. The first one is just that used by Sanchez et al. in a recent paper on holographic materials, where the incorporation of $\mathrm{TiO}_{2}$ nanoparticles provides $\Delta n$ greater than $10^{-2}$ (see Supporting Information for experimental details). ${ }^{[6]}$ The scattering coefficients $a$ found for samples $\mathrm{Zr} 0$ and $\mathrm{Zr} 1$ are $2 \times 10^{-3}$ and $1.2 \times 10^{-3} \mu \mathrm{m}^{-1}$, respectively. Note that, for a material of $15 \mu \mathrm{m}$ thickness, these values would correspond to ca. $3 \%$ ( $\mathrm{Zr} 0)$ and ca. $1.8 \%(\mathrm{Zr} 1)$ scattered light, which is about eightfold noise reduction compared to that reported for holographic materials based on $\mathrm{TiO}_{2}$ nanoparticles. To corroborate further the low level of scattering, we performed a second experiment previously used for characterization of Aprilis ULSH photopolymers. ${ }^{[15]}$ When the Zr1 sample is exposed to a single recording beam, there is virtually no transmittance degradation (Fig. 4), indicating that the scattering is very weak, despite the incorporation of HRIS. This very weak character of the noise grating was also confirmed by the noise grating angular selectivity measurement shown in Figure 4. In the presence of the noise grating a dip in diffraction efficiency is expected near the Bragg resonance, which is absent in our 


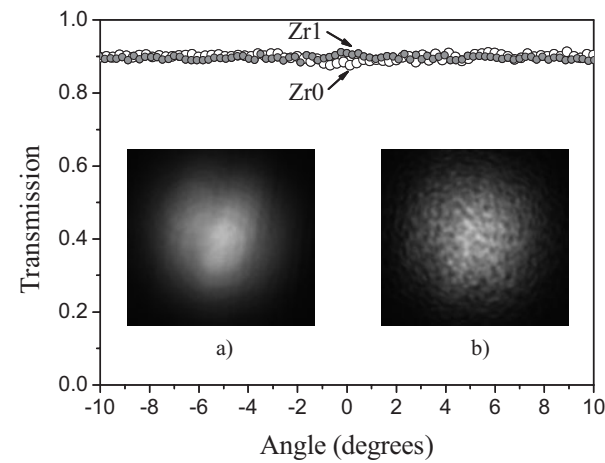

Figure 4. Noise grating angular selectivity measurement for samples $\mathrm{ZrO}$ and $\mathrm{Zr} 1$. The samples were exposed and read-out by a coherent s-polarized single beam of $532 \mathrm{~nm}$ wavelength and $5 \mathrm{~mW} \mathrm{~cm}$ intensity. The insets show the beam profiles transmitted through sample $\mathrm{Zr} 1$ a) before and b) after exposure to ca. $15 \mathrm{~J} \mathrm{~cm}^{-2}$.

samples. The insets in Figure 4 show the beam passing through the sample before (inset a) and after (inset b) exposure (exposure energy is $15 \mathrm{~J} \mathrm{~cm}^{-2}$ ). Some granularity observed in inset $\mathrm{b}$ is due to the speckle. Experiments performed with p-polarized writing beams show identical results.

In summary, we have shown that incorporation of Zr-based HRIS in a photopolymerizable volume holographic recording sol-gel material results in an increase in refractive index modulations up to the $10^{-2}$ range and diffraction efficiencies near $100 \%$. The index modulation is similar to recently reported photopolymers with $\mathrm{TiO}_{2}$ nanoparticles as the HRIS, but scattering is markedly reduced (ca. eightfold) as a consequence of the molecular rather the nanoparticle nature of our HRIS. Though the present work has been based on sol-gel materials, we suggest that the same approach can be used to enhance the dynamic range of conventional holographic photopolymers.

\section{Experimental}

Sample Preparation: A silica sol was prepared by acid hydrolysis (21.3 mmol $\mathrm{H}_{2} \mathrm{O} / \mathrm{HCl}, \mathrm{pH}$ 2) of glycidoxypropyltrimethoxysilane (GPTMS, IUPAC name trimethoxy-[3-(oxiran-2-ylmethoxy)propyl]silane; $11.8 \mathrm{mmol}$, from Aldrich) and tetraethylorthosilicate (TEOS; $1.8 \mathrm{mmol}$, from Aldrich), under vigorous stirring. The GPTMS/TEOS molar ratio is thus selected to minimize binder shrinkage upon holographic recording [16]. The silicon alkoxide molarities are thus selected to ensure all water is consumed $(11.8 \times 1.5+1.8 \times 2)$ before zirconium isopropoxide is added. After about $10 \mathrm{~min}$, a solution of IRGACURE784 (0.1 mmol, from Ciba-Geigy) in POEA ( $3.1 \mathrm{mmol}$, from Aldrich) was added, and after another $10 \mathrm{~min}$ a solution of zirconium isopropoxide isopropanol complex $\left(\mathrm{Zr}\left(\mathrm{O}^{i} \mathrm{Pr}\right){ }_{4}{ }^{i} \mathrm{PrOH} ; 1.5 \mathrm{mmol}\right.$, from $\mathrm{Al}-$ drich) in methacrylic acid (MA; $7 \mathrm{mmol}$, from Aldrich) was added to the mixture, still under vigorous stirring. The solution was stirred for another $5 \mathrm{~min}$ and then filtered with a $0.2 \mu \mathrm{m}$ Millipore filter. Films of thickness ca. $35 \mu \mathrm{m}$ were obtained by immediately casting the filtered solution on glass microscope slides. Samples were left to dry in the dark at room temperature for 5 days prior to hologram recording.

Sample Characterization: The holographic gratings were recorded in the material by the interference of two coherent s-polarized beams of a frequency-doubled Nd:YAG laser with a wavelength of $532 \mathrm{~nm}$ and working in the single longitudinal-mode regime. The intensity ratio between the two recording beams was $r=0.5$. The recording waves were incident on the sample in an unslanted configuration, intersecting the plate holder position at an angle of $7.9^{\circ}$ with respect to the sample normal, producing interference fringes of spatial frequency ca. 500 lines $\mathrm{mm}^{-1}$. The grating growth was monitored in real time by a non-actinic probe beam of wavelength $632.8 \mathrm{~nm}$ and $0.5 \mathrm{~mW}$ power from a He-Ne laser (Bragg angle ca. $\left.9.45^{\circ}\right)$. The sample was placed on a precision rotation stage to allow the Bragg angle of the probe beam to be adjusted and the grating angular selectivity to be measured. SEM and EDX were performed on carbon-coated samples using a Zeiss DSM-950 instrument. SFM topographic images were acquired under ambient conditions with a microscope head (control unit and software from Nanotec Electronica Inc., www.nanotec.es). Rectangular cantilevers with a nominal force constant of $11 \mathrm{~N} \mathrm{~m}^{-1}$ and ultra-sharp Si tips (from NT-MDT) were used in the tapping operation mode.

Received: December 13, 2005

Final version: February 4, 2006

[1] a) M. Haw, Nature 2003, 422, 556. b) S. J. Zilker, ChemPhysChem 2002, 3, 333

[2] D. H. Close, A. D. Jacobson, J. D. Margerum, R. G. Brault, F. J. McClung, Appl. Phys. Lett. 1969, 14, 159

[3] a) J. Ashley, M.-P. Bernal, G. W. Burr, H. Coufal, H. Guenther, J. A. Hoffnagle, C. M. Jefferson, B. Marcus, R. M. Macfarlane, R. M. Shelby, G. T. Sincerbox, IBM J. Res. Dev. 2000, 44, 341. b) M. L. Schilling, V. L. Colvin, L. Dhar, A. L. Harris, F. C. Schilling, H. E. Katz, T. Wysocki, A. Hale, L. L. Blyler, C. Boyd, Chem. Mater. 1999, 11, 247. c) R. Changkakoti, L. R. B. Patterson, C. Dreze, A. K. Ghosh, Proc. SPIE 1995, 2482, 213. d) V. Weiss, A. A. Friesem, V. A. Krongauz, J. Imaging. Sci. Technol. 1997, 41, 371. e) B. M. Monroe, E. K. Smothers, in Polymers for Light Wave and Integrated Optics, Part 1: Foundations (Ed: L. A. Hornak) Marcel Dekker, New York 1992, p. 145. f) D. A. Waldman, R. T. Ingwall, P. K. Dhal, M. G. Horner, E. S. Kolb, H.-Y. S. Li, R. A. Minns H. G. Schild, Proc. SPIE 1996, 2689, 127. g) A. Fimia, N. López, F. Mateos, R. Sastre, J. Pineda, F. Amat-Guerri, Appl. Opt. 1993, 32, 3706. h) L. Carretero, A. Murciano, S. Blaya, M. Ulibarrena, A. Fimia, Opt. Express 2004, 12, 1780.

[4] W. S. Colburn, K. A. Haines, Appl. Opt. 1971, 10, 1636.

[5] a) B. M. Monroe, W. K. Smothers, D. E. Keys, R. R. Krebs, D. J. Mickish, A. F. Harrington, S. R. Schicker, M. K. Armstrong, D. M. T. Chan, C. I. Wheathers, J. Imaging Sci. 1991, 35, 19. b) T. J. Trout, J. J. Schmieg, W. J. Gambogi, A. M. Weber, Adv. Mater. 1998, $10,1219$.

[6] a) N. Suzuki, Y. Tomita, Appl. Opt. 2004, 43, 2125. b) N. Suzuki, Y. Tomita, T. Kojima, Appl. Phys. Lett. 2002, 81, 4121. c) C. Sanchez, M. J. Escuti, C. van Heesch, C. W. M. Bastiaansen, D. J. Broer, J. Loos, R. Nussbaumer, Adv. Funct. Mater. 2005, 15, 1623.

[7] P. W. Oliveira, H. Krug, P. Müller, H. Schmidt. Mater. Res. Soc. Symp. Proc. 1996, 435, 553.

[8] E. A. Chandross, W. J. Tomlinson, G. D. Aumiller. Appl. Opt. 1978, $17,566$.

[9] N. F. Borelli, D. L. Morse, Appl. Phys. Lett. 1983, 43, 992.

[10] V. I. Sukhanov, M. V. Kazankova, A. M. Kursakova, O. V. Andreeva, Opt. Spectrosc. 1988, 65, 282.

[11] P. Cheben, T. Belenguer, A. Nunez, D. Levy, F. del Monte, Opt. Lett. 1996, 21, 1857.

[12] a) P. Cheben, M. L. Calvo, Appl. Phys. Lett. 2001, 78, 1490. b) F. del Monte, G. Ramos, T. Belenguer, E. Bernabeu, D. Levy, Proc. SPIE 2002, 4802, 51.

[13] H. Kogelnik, Bell. Syst. Tech. J. 1969, 48, 2909.

[14] M. G. Schnoes, L. Dhar, M. L. Schilling, S. S. Patel, P. Wiltzius, Opt Lett. 1999, 24, 658.

[15] J. A. Frantz, R. K. Kostuk, D. A. Waldman, Proc. SPIE 2001, 4296, 159.

[16] G. Ramos, A. Alvarez-Herrero, T. Belenguer, F. del Monte, D. Levy, Appl. Opt. 2004, 43, 4018. 